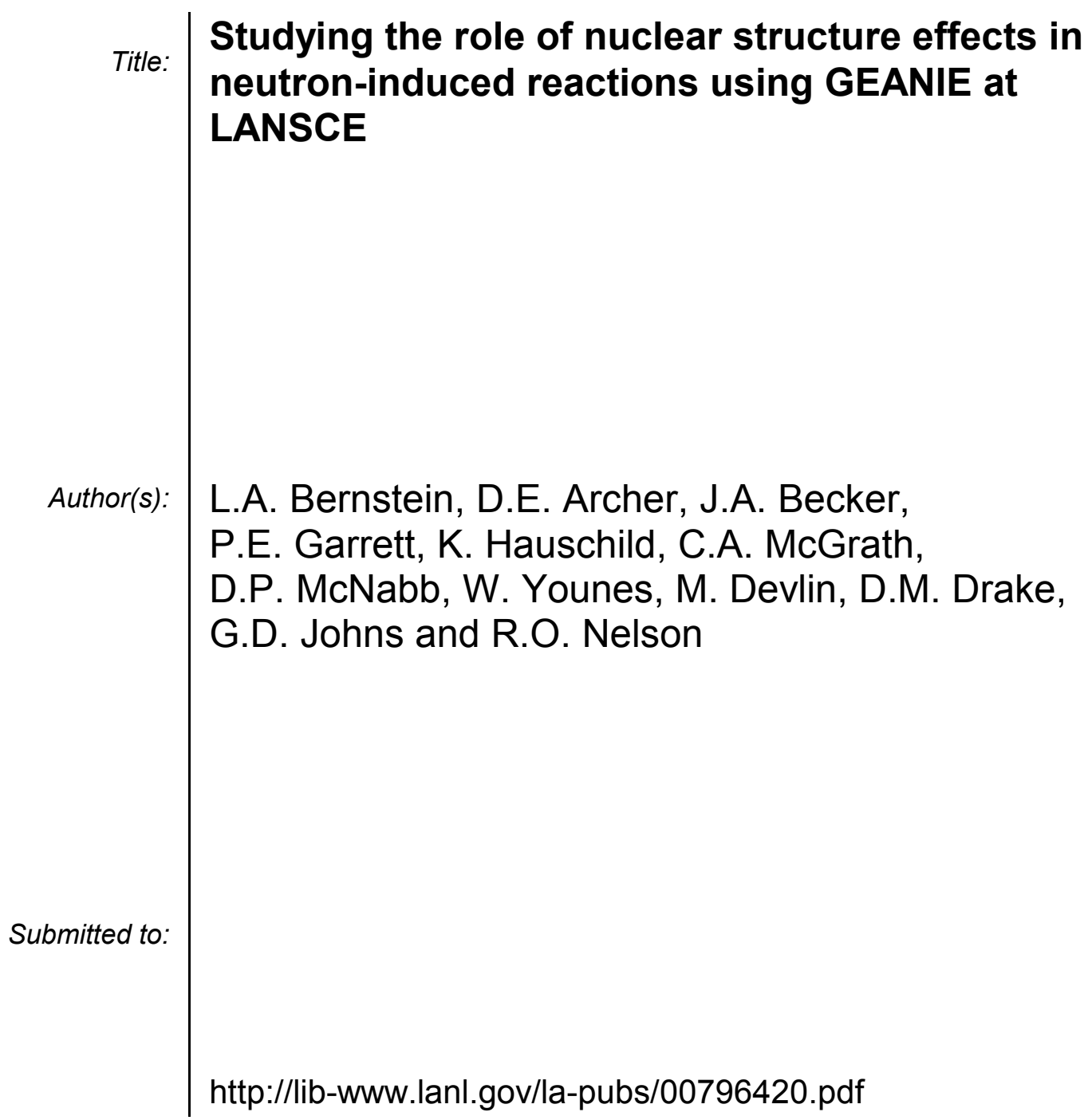

Los Alamos National Laboratory, an affirmative action/equal opportunity employer, is operated by the University of California for the U.S. Department of Energy under contract W-7405-ENG-36. By acceptance of this article, the publisher recognizes that the U.S. Government retains a nonexclusive, royaltyfree license to publish or reproduce the published form of this contribution, or to allow others to do so, for U.S. Government purposes. Los Alamos National Laboratory requests that the publisher identify this article as work performed under the auspices of the U.S. Department of Energy. Los Alamos National Laboratory strongly supports academic freedom and a researcher's right to publish; as an institution, however, the Laboratory does not endorse the viewpoint of a publication or guarantee its technical correctness. 


\title{
Studying the role of nuclear structure effects in neutron-induced reactions
} using GEANIE at LANSCE

\author{
L.A. Bernstein ${ }^{a}$, D.E. Archer ${ }^{a}$, J.A. Becker ${ }^{a}$, P.E. Garrett ${ }^{a}$, K. Hauschild ${ }^{a}$, C.A. McGrath ${ }^{a}$, \\ D.P. McNabb ${ }^{a}$, W. Younes ${ }^{a}$, M. Devlin ${ }^{b}$, D.M. Drake ${ }^{b}$, G.D. Johns ${ }^{b}$, R.O. Nelson ${ }^{b}$ \\ ${ }^{a}$ Lawrence Livermore National Laboratory, Livermore, CA 94551 USA \\ ${ }^{b}$ Los Alamos National Laboratory, Los Alamos, NM 87545 USA
}

The GEANIE (GErmanium Array for Neutron Induced Excitations) spectrometer at the LANSCE/WNR (Los Alamos Neutron Science Center/Weapons Neutron Research) 800 $\mathrm{MeV}$ spallation neutron source provides a unique tool for studying reaction dynamics for incident particle energies up to and beyond $250 \mathrm{MeV} / \mathrm{amu}$. In this paper we will discuss several of the recent results from GEANIE and their implications for reaction dynamics modeling.

\section{INTRODUCTION}

Efforts to model and measure absolute neutron-induced reaction cross sections have for the most part been limited by experimental techniques to neutron energies found in reactors and light-ion fusion reactions (i.e., $t(d, n) \alpha$ etc.). This can in large part be attributed to the difficulty involved in making high-flux mono-energetic neutron beams with $E_{n}>14 \mathrm{MeV}$. The result has been a lack of guidance for (n,xnypza) reaction modeling for $E_{n}>1-2 \mathrm{MeV}$. These limitations become particularly exacerbated in nuclei where structure effects (i.e., near shell closures, high deformation etc.) or fission complicates the models. The GEANIE spectrometer at LANSCE/WNR fills this gap in experimental technique by allowing for efficient measurement of neutron-induced $\gamma$-ray partial cross sections over a wide range of incident neutron energies $\left(1<\mathrm{E}_{\mathrm{n}}(\mathrm{MeV})<250\right)$. GEANIE consists of 20 Comptonsuppressed and 6 unsuppressed HPGe detectors located at the $60^{\circ}$ right beam line at the LANSCE/WNR spallation source. Eleven of the GEANIE detectors are Low Energy Planar Spectrometers (LEPS) with excellent timing and energy resolution. LANSCE/WNR provides a white source of neutrons through the spallation of a tungsten target with an 800 $\mathrm{MeV} 2-5 \mu \mathrm{A}$ beam of protons. The neutron energy is determined by the time-of-flight technique. Early results from GEANIE [1] highlighted the deficiencies in the modeling of neutron-induced reaction modeling on a ${ }^{196} \mathrm{Pt}$ target. More recently, measurements on a ${ }^{92}$ Mo target have identified $\gamma$-rays in over 2 dozen reaction products [2]. In this paper we 
will present an overview of several recent results from GEANIE, including data on a ${ }^{92} \mathrm{Mo}$ and two Actinide $\left({ }^{235} \mathrm{U}\right.$ and $\left.{ }^{239} \mathrm{Pu}\right)$ targets. The implications of these results for reaction modeling from low too high $\left(\mathrm{E}_{\mathrm{n}}<250 \mathrm{MeV}\right)$ energy will be presented and plans for future experiments discussed.

\section{EXPERIMENTAL SET-UP}

GEANIE is currently comprised of 9 Compton suppressed and 6 unsuppressed coaxial Ge detectors with approximately $25 \%$ of the efficiency of a 3" X 3" $\mathrm{NaI}$ crystal and 11 Compton suppressed planar Ge detectors (LEPS - Low Energy Photon Spectrometers). GEANIE is located $20.34 \mathrm{~m}$ from the LANSCE/WNR spallation neutron source on the $60^{\circ}$ right flight path. Neutron energies at the WNR "white" source cover the range from less than $1 \mathrm{MeV}$ to $\approx 600 \mathrm{MeV}$. Neutron fluences on target were measured with a ${ }^{235} \mathrm{U} /{ }^{238} \mathrm{U}$ fission chamber [3] located $18.48 \mathrm{~m}$ from the center of the spallation target. Experiments are performed by exposing a targets in the neutron beam and recording the $\gamma$-ray energy (pulse height) and the neutron time-of-flight (TOF).

Spallation neutrons were produced at the ${ }^{\text {nat }} \mathrm{W}$ spallation target irradiated by an $800 \mathrm{MeV}$ proton beam with an average current of $\approx 2 \mu \mathrm{A}$ during the 1998 experiments and $\approx 6 \mu \mathrm{A}$ during the 1999 run. The neutron flux decreases by approximately a factor of 5 between 5 and $20 \mathrm{MeV}$. The beam time structure consisted of a train of $1.8 \mu$ s micropulses (referred to as a "macropulse") at a typical repetition rate of $120 \mathrm{~Hz}$. In-beam recorded data consisted of $\gamma$-ray pulse heights with $20 \mathrm{keV} \leq \mathrm{E}_{\gamma} \leq 1 \mathrm{MeV}$ for the LEPS and $50 \mathrm{keV} \leq \mathrm{E}_{\gamma} \leq 4 \mathrm{MeV}$ for the coaxial detectors together with the event time, correlated to the beam micropulse RF signal. Beam-off recorded data included the same $\gamma$-ray pulse height information together with a 100 ns-per-tick "long-range clock" that was reset at the beginning of every macropulse. The neutron encrgy of an cvent that produccd a specific detected in-beam $\gamma$-ray was obtained from the difference in the time of arrival of the $\gamma$-ray the " $\gamma$-flash" coming from the spallation target. This is referred to as the time-of-flight (TOF) technique. Data were recorded onto magnetic tape using a specially modified version of the VME-based Michigan State University data acquisition system [4].

\section{EXPERIMENTS}

\subsection{The ${ }^{92}$ Mo experiment}

The capabilities of the array to study non-yrast structure and investigate reaction dynamics of high-energy neutron beams in the $A=90$ region was demonstrated in an experiment performed at GEANIE using a $13 \mathrm{~g}$ sample of highly enriched ${ }^{92} \mathrm{Mo}$. The inbeam data were sorted into $\mathrm{E}_{\gamma}$ vs. Time-of-Flight matrices for both the planar and the coaxial Ge detectors. $\gamma-\gamma$ coincidence matrices were also sorted. A significant amount of new spectroscopy was performed and the results published [5]. 


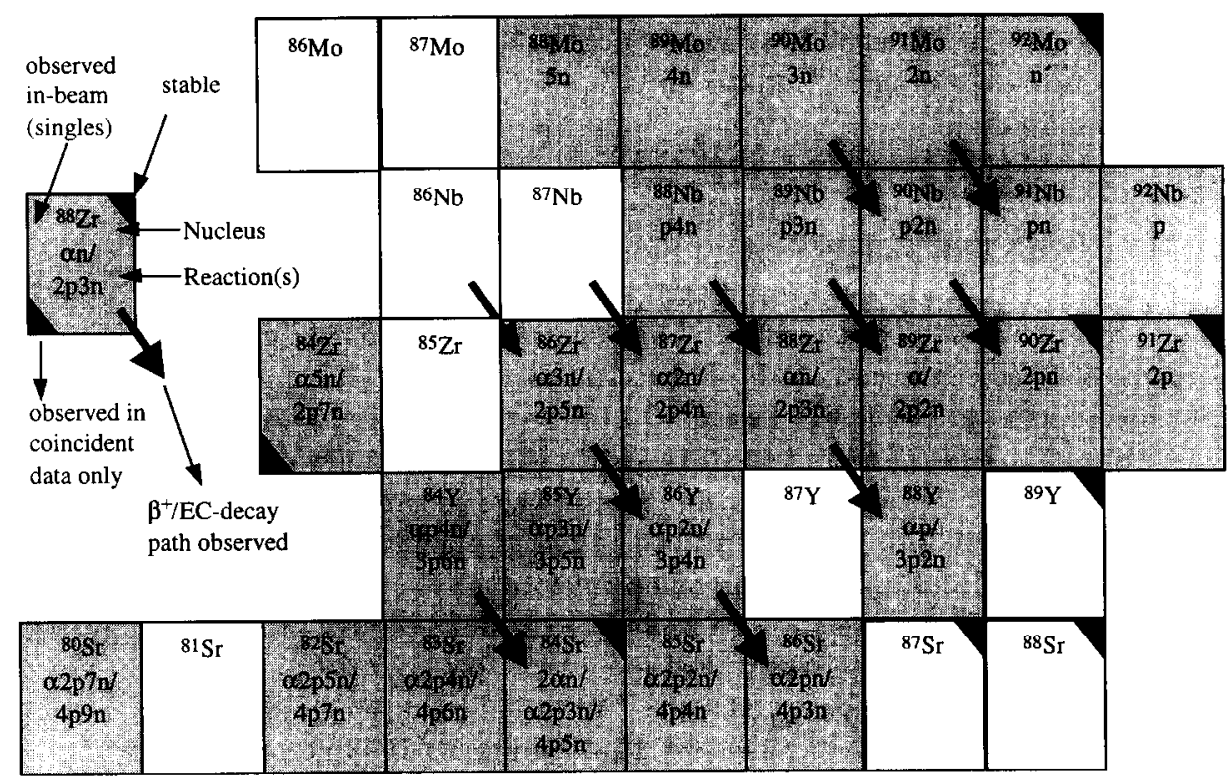

Figure 1. A partial chart of the nuclides showing the products bbserved following the bombardment of a target of ${ }^{92} \mathrm{Mo}$ with high-energy neutrons.

The investigation of reaction dynamics involved constructing excitation functions of $\gamma$ rays in the residual nuclei. The exit channels were identified using the $\gamma-\gamma$ coincidences and the reaction thresholds from the $\gamma$-ray yields. Excitation functions for neutron energies up to $250 \mathrm{MeV}$, normalized at one energy to the calculated cross section for the $2^{+} \rightarrow 0^{+}$transition in ${ }^{92} \mathrm{Mo}$, were extracted for a total of 26 different isotopes, as displayed in Figure 1. The lightest isotope observed was ${ }^{80} \mathrm{Sr}$ via the $(\mathrm{n}, \alpha 2 \mathrm{p} 7 \mathrm{n} / 4 \mathrm{p} 9 \mathrm{n})$ reaction channel. The experimental data were compared with the results of GNASH calculations for the partial $\gamma$ ray cross sections. The calculations include compound nucleus, pre-equilibrium, multiplepre-equilibrium, and direct reaction processes.

When all the data are viewed together, a consistent picture emerges as to the strengths and weaknesses in the present reaction cross section calculations. Below $\sim 20 \mathrm{MeV}$, the calculations overall do a reasonably good job of reproducing the cross section to the various final channels. Problems can arise, however, from weaknesses in modeling the $\gamma$-ray cascade. This is especially apparent in the case of ${ }^{91} \mathrm{Nb}$, shown in figure 2 . The predicted cross section for the positive-parity levels is in good agreement with the data, whereas that for the negative-parity states is not.

The nuclear level density in both the compound and residual nuclei is a critical input parameter for Hauser-Feshbach based reaction model codes. The level density for the compound system is often known for nuclei near stability due to neutron resonance studies. 


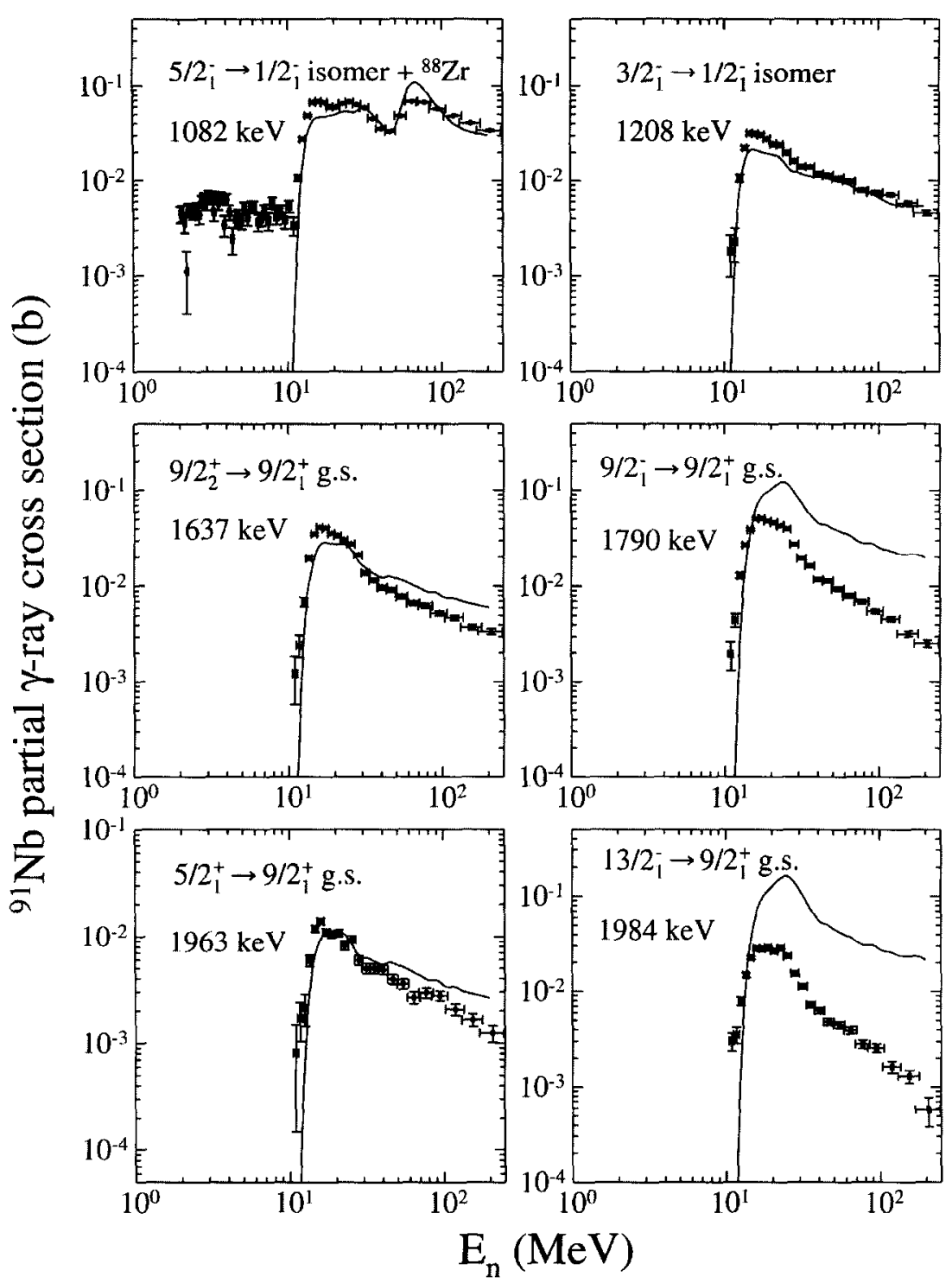

Figure 2. Excitation functions for $\gamma$-rays originating from the ${ }^{92} \mathrm{Mo}(\mathrm{n}, \mathrm{pn} \gamma)^{91} \mathrm{Nb}$ reaction. While the calculated cross sections for the transitions from the positive parity states agree with the data, those for the negative parity, especially high-spin levels do not. 


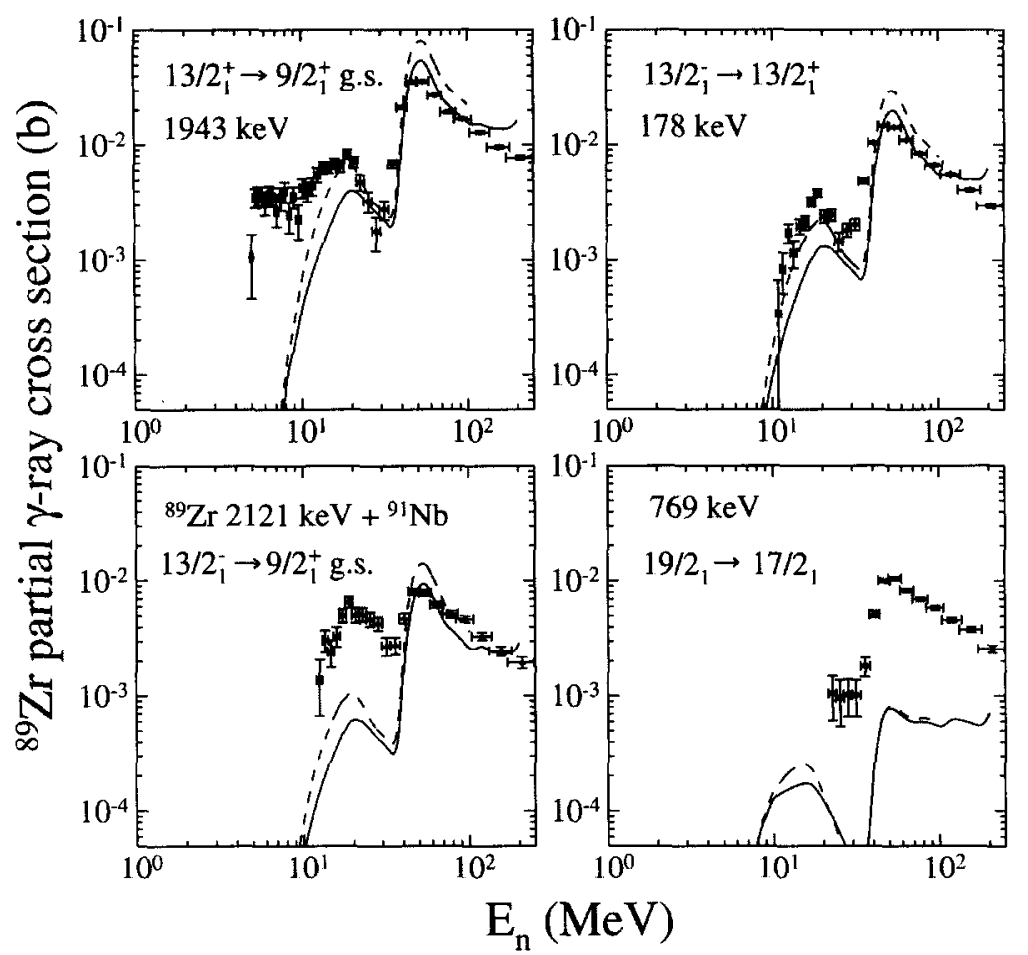

Figure 3. Excitation functions for $\gamma$-rays originating from the ${ }^{92} \mathrm{Mo}(\mathrm{n}, \alpha 2 \mathrm{p} 2 \mathrm{n} \gamma)^{89} \mathrm{Zr}$ reaction. The difference in threshold energies due to the $\alpha$ binding energy provides a clear distinction between $\alpha$-emission and the $2 \mathrm{p} 2 \mathrm{n}$ process. The $2121-\mathrm{keV}$ transition is unresolved from a ${ }^{91} \mathrm{Nb} \gamma$-ray, but it is believed to contribute less than $10 \%$ of the cross section above $30 \mathrm{MeV}$. The solid curves are model predictions obtained using level density parameters obtained from systematics, whereas the dashed curves are predictions where the level density parameters have been "tuned" to improve the agreement with the $(n, \alpha)$ process.

The situation is often far more ambiguous for the product nuclei, resulting in the use of systematics to obtain the appropriate level density to use in a model calculation. The effect of changing the level density parameter is shown in figure 3 where the excitation functions for $\gamma$-ray originating from the ${ }^{92} \mathrm{Mo}(\mathrm{n}, \alpha / 2 \mathrm{p} 2 \mathrm{n}){ }^{89} \mathrm{Zr}$ reaction are displayed. The solid curves are GNASH [6] calculations performed by $\mathrm{M}$. Chadwick at LANL using the default level density parameters. The level density parameters were then adjusted in order to improve the predictions of the model for the $(\mathrm{n}, \gamma)$ process. The dashed lines in figure 3 show these calculations. While there is an improvement in the $(n, \gamma)$ channel it comes at the expense of the (n.2p2n) channel. 


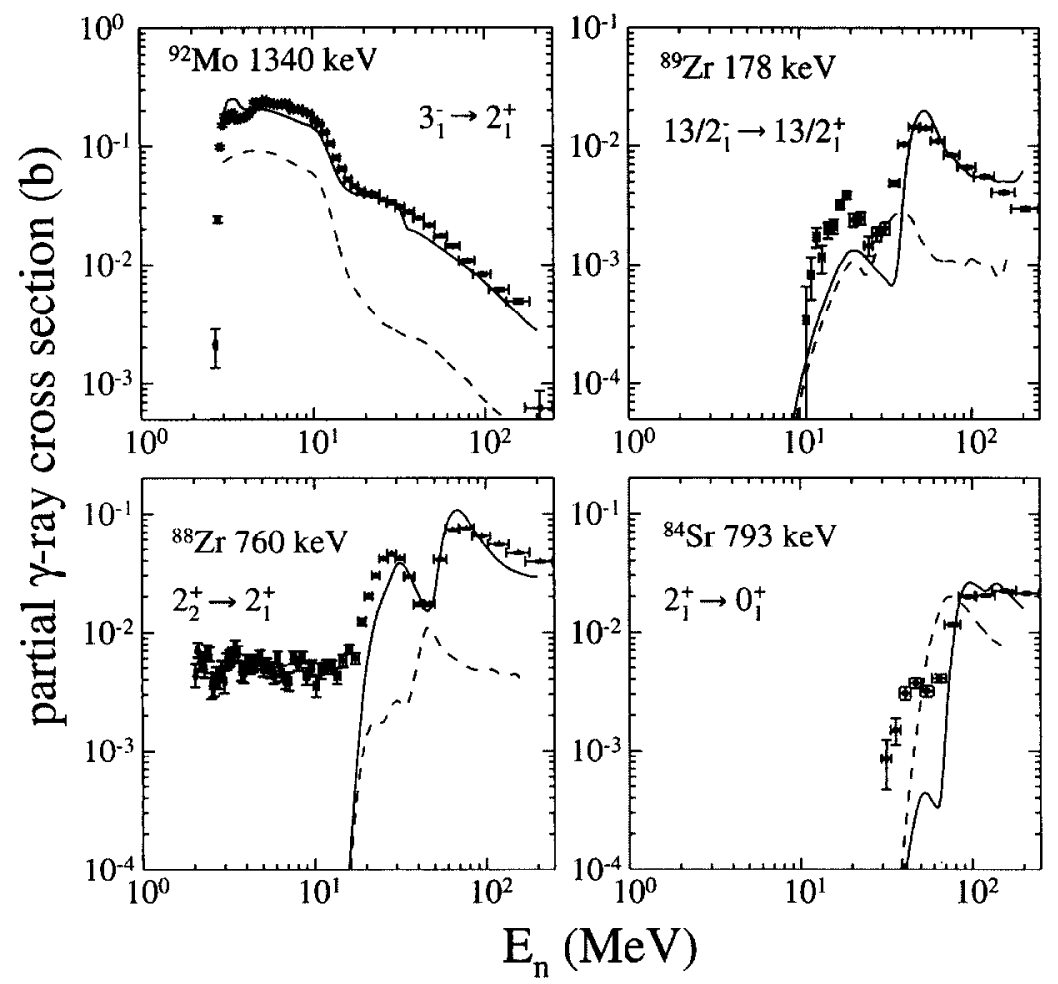

Figure 4. Excitation functions for several $\gamma$-rays highlighting the improvements in model calculations made during the course of this work. The first panel shows the ${ }^{92} \mathrm{Mo} 3_{1}^{-} \rightarrow 2_{1}^{+}$ partial cross section predicted before (dashed) and after (solid) an improved level scheme was used as input. The remaining panels show the result of code "de-bugging" (dashed curve - before, solid curve - after).

A substantial benefit has come about from the close collaboration of theory and experiment. Figure 4 shows a remarkable improvement in the model calculations from the initial iteration (dashed curve) to the final calculation (solid curve) where both the level schemes used in the calculation were modified to include the new levels established by this work and several "bugs" in the coding were eliminated. Without the interrogation of the model output provided by the experimental data these errors would not have been detected.

A somewhat surprising capability of the array is provided by the ability to measure $\gamma$-rays between the beam burst. Very long-lived isomeric states, on the order of $100 \mu \mathrm{s}$ to $10 \mathrm{~ms}$, can be observed to decay during the beam-off period and their lifetime can be determined. For shorter lived isomeric states, less than several hundred ns, it is possible to extract their decay curves for times that correspond to neutron energies below their reaction threshold. An example of this is shown in Figure 5. 

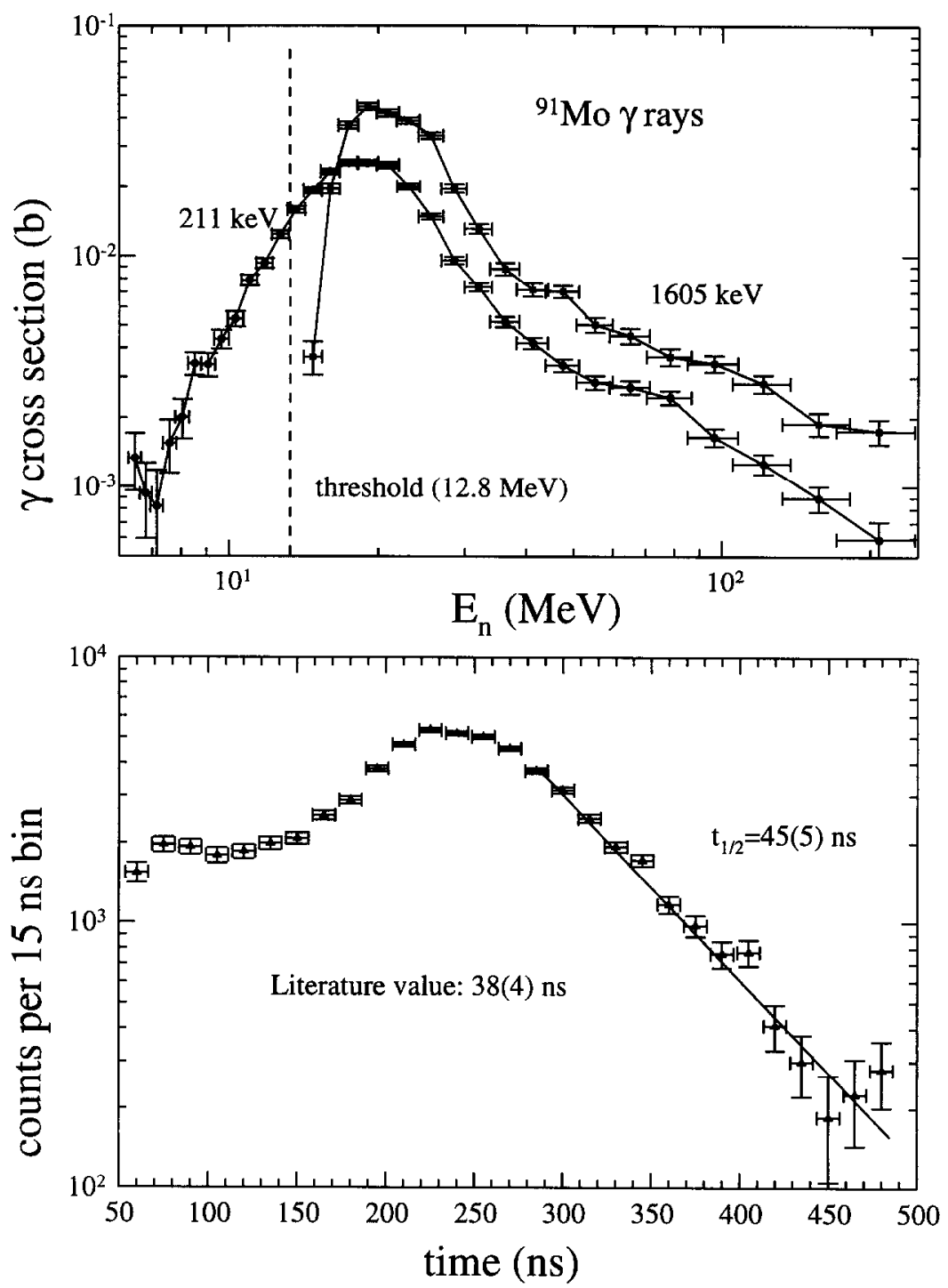

Figure 5. An example of the ability to determine life times of isomeric states by extracting their decay curves below the production threshold. The lop panel shows the effect of an isomer on a transition (211 keV) compared to a prompt transitions (1605 keV). The bottom panel shows the half-life extracted from the $211 \mathrm{keV}$ transition. 


\subsection{The ${ }^{239}$ Pu experiments}

The ${ }^{92}$ Mo experiment highlight the capability of GEANIE to use partial $\gamma$-ray cross sections to gain insight into the various reaction mechanisms that take place at different incident projectile energies. GEANIE experiments also offer the possibility of inferring total channel cross sections individually from partial $\gamma$-ray cross sections. Such an effort involves both experiment (in the form of measured $\gamma$-ray partial cross sections, well-characterized $\gamma$ ray efficiencies, etc.) as well as theory to relate the partial cross sections to the total channel cross section.

An attempt was made to carry this out for the $(n, 2 n)$ reactions on a ${ }^{239} \mathrm{Pu}$ target at GEANIE in 1998 and 1999. These experiments pushed the limits of experiment at GEANIE. The 4.5 gram Plutonium target was highly radioactive leading to a large $\gamma$-ray background. In addition, sizable discrete and statistical $\gamma$-ray contamination was present from neutroninduced fission. Two different thickness targets were used; 10 mil (thin) and 20 mil (thick). A detailed efficiency calibration for these targets was performed using a combination of point and distributed source $\gamma$-ray calibration measurements [7].

The modeling effort included two Hauser-Feshbach based reaction models with preequilibrium and fission competition added: the GNASH model [6] from M. Chadwick at Los Alamos National Laboratory and the IDA model from H. Chen and W.E. Ormand at LLNL. Two different GNASH calculations were made. The first calculation (GNASH 99a) used a ${ }^{238} \mathrm{Pu}$ level scheme from ENSDF. The second GNASH (GNASH 00b) and the IDA calculations used an "augmented" level scheme where levels were added based on a comparison with the isotone ${ }^{236} \mathrm{U}$ and the isotope ${ }^{240} \mathrm{Pu}$.

A total of 8 transitions in ${ }^{238} \mathrm{Pu}$ were identified in the data. Partial $\gamma$-ray cross sections were measured and compared with the results of the GNASH and IDA calculations. The reaction cross section in the GNASH 00b calculations was adjusted in order to fit the magnitude of the yrast $6^{+} \rightarrow 4^{+}$cross section. In general, although good agreement is seen between the measured and calculated cross sections, there is some disagreement near threshold $\left(E_{n} \approx 6-8 \mathrm{MeV}\right)$ and at higher $\left(E_{n}>14 \mathrm{MeV}\right)$ incident neutron energy.

Several possible approaches exist for extracting the $(n, 2 n)$ cross section from the partial $\gamma$-ray cross sections. The first approach would be to rely solely on the lowest transition from the ground state band and to infer the total channel cross section using the relation:

$$
\sigma^{\text {deduced }}(n, 2 n)=\sigma^{\text {(theory) }}(n, 2 n) \times \frac{\sigma^{(\text {exp })}\left(6_{1}^{+} \rightarrow 4_{1}^{+}\right)}{\sigma^{\text {(theory) }}\left(6_{1}^{+} \rightarrow 4_{1}^{+}\right)}
$$

The success of this approach is very dependent on the details of the low-lying level scheme in the residual nucleus since the entire channel cross section is inferred from a single transition. A different approach that reduces this dependence is to combine all of the partial cross sections that feed the ground state of the reaction product from "parallel" paths. Only non-coincident $\gamma$-rays are added together to insure that the $\gamma$-ray intensity is not doublecounted:

$$
\sigma^{\text {deduced }}(n, 2 n)=\sigma^{(\text {theory) }}(n, 2 n) \times \sum_{\text {parallel }} \frac{\sigma_{i}^{(\text {exp })}\left(\gamma_{i}\right)}{\sigma^{(\text {theory) }}\left(\gamma_{i}\right)}
$$




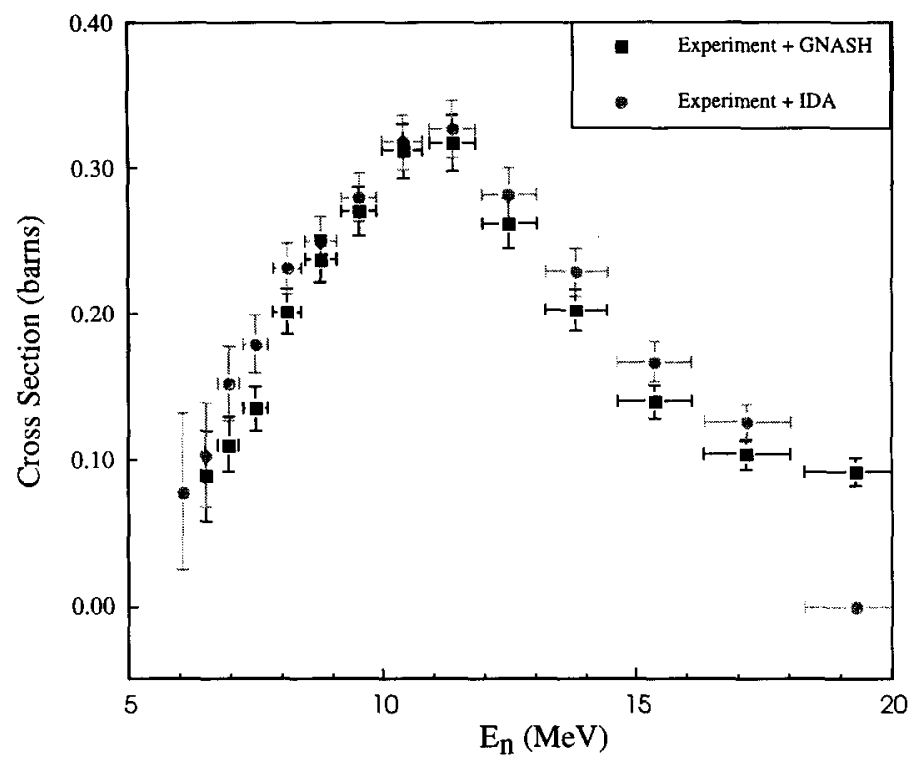

Figure 6. The ${ }^{239} \mathrm{Pu}(\mathrm{n}, 2 \mathrm{n})$ cross section extracted from the GEANIE data combined with the results of the GNASH (black squares) and IDA (gray circles) model calculations.

Figure 6 shows the extracted $(n, 2 n)$ cross sections using both GNASH and the IDA calculations. The results are remarkably similar; thereby lending confidence to the proposition that GEANIE can not only be used to probe reaction mechanisms, but also to measure channel cross sections indirectly.

\subsection{The ${ }^{235} \mathrm{U}$ experiment}

In addition to providing insight into compound and pre-compound reaction dynamics GEANIE offered a unique opportunity to measure the neutron-induced fission process over a wide range of incident neutron energies. There is remarkably little experimental information regarding prompt fission fragment distributions for incident neutron energies other than thermal or $14 \mathrm{MeV}$. Most of the measurements are of "cumulative yields" following $\beta$-decay of the fission fragments back towards the valley of stability. This process results in a loss of information regarding the prompt yields.

A total of three experiments on a ${ }^{235} \mathrm{U}$ target were carried out at GEANIE in parallel with the ${ }^{239} \mathrm{Pu}$ experiments. Cross sections for even-even fission fragments, confirmed as being uncontaminated using the $\gamma-\gamma$ coincidence data, were obtained from the $\gamma$-singles data. A total of 32 transitions from ${ }^{235} \mathrm{U}(\mathrm{n}, \mathrm{f})$ were measured for $0<\mathrm{E}_{\mathrm{n}}(\mathrm{MeV})<250$. The details of these experiments are reported in [8-9]. Figure 7 shows a map of the relative fission yields for these nuclei for $E_{n}=14 \mathrm{MeV}$. 

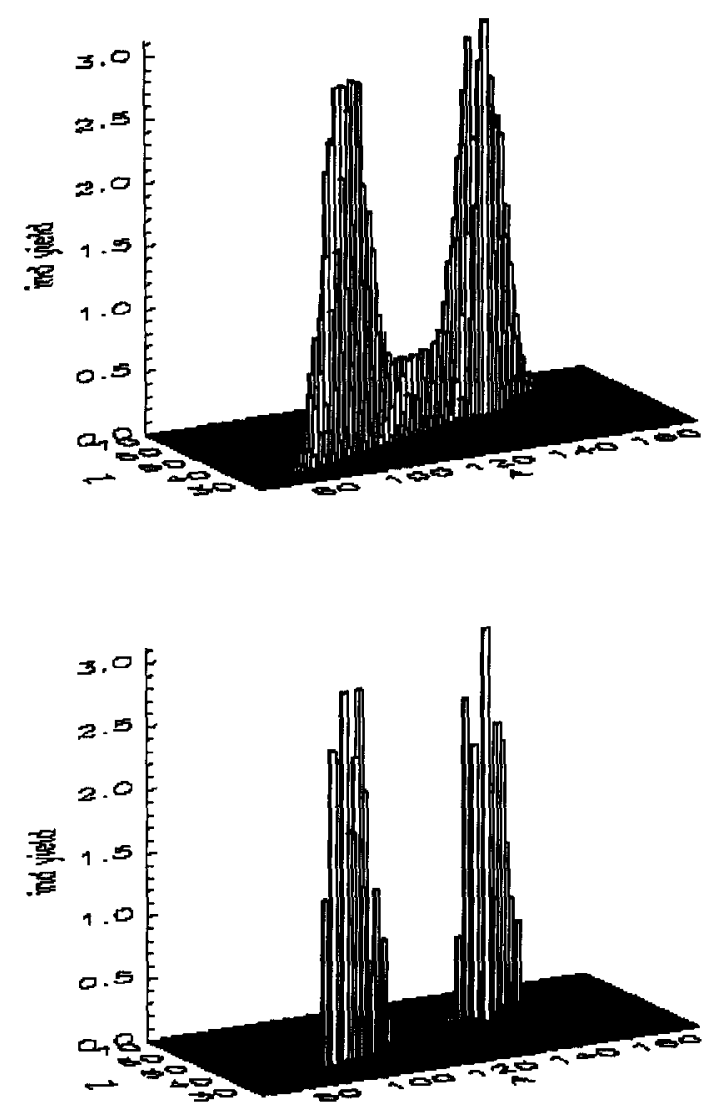

Figure 7. Extracted independent fission fragment yields for ${ }^{235} U(n, f)$. The top panel is from a compilation for $14 \mathrm{MeV}$ neutrons from England and Rider. The bottom panel shows the yield for 32 even-even fission fragments resulting from fission caused by $14 \mathrm{MeV}$ neutrons from the 1999 GEANIE data. The $\mathrm{x}$ - and $\mathrm{y}$-axes are mass and element numbers respectively.

\section{CONCLUSIONS}

The GEANIE spectrometer at LANSCE/WNR offers a wide range of experimental insight into reaction dynamics modeling and measurements for neutron-induced reactions over a wide range of incident neutron energies. One of the capabilities presented in this paper includes using the population of low-lying states in residual nuclei to gain insight into the population mechanisms taking place in the nuclear reaction. This ability was shown in 
the ${ }^{92}$ Mo experiment. Deficiencies in the modeling of the $\gamma$-ray cascade were evident by examining the population of negative parity states in ${ }^{91} \mathrm{Nb}$.

The experiments using a ${ }^{239} \mathrm{Pu}$ target represent some of the difficulties involved in making measurements on highly radioactive targets. They also serve to illustrate the ability of the array to extract channel cross sections based on partial $\gamma$-ray cross sections and model calculations. Although individual $\gamma$-ray cross sections are not well predicted by reaction models sums of $\gamma$-rays can be used to eliminate some of these uncertainties.

Finally, the ${ }^{235} \mathrm{U}$ experiment demonstrated the unique expcrimental insight that GEANIE can provide into the process of neutron-induced fission. Future experiments in this vein involve measuring fission fragment cross section in sub-actinide nuclei such as Bismuth and Lead as well as other threshold-fission systems

This work has been performed under the auspices of the U.S. Department of Energy by Lawrence Livermore National Laboratory under contract No. W-7405-ENG-48 and Los Alamos National Laboratory under contract No. W-4705-ENG-36.

\section{REFERENCES}

1. L.A. Bernstein et al., Phys. Rev. C57, R2799 (1998).

2. P.E. Garrett et al., Phys. Rev. C 62, 054608 (2000).

3. S.A. Wender et al., Nucl. Instrum. and Meth. A336, 2 (1993).

4. M. Maier, M. Robertson, A. Vander Molen, and G.D. Westfall, NIM A337, 619 (1994).

5. P.E. Garrett et al., Phys. Rev. C62, 014307 (2000).

6. P.G. Young, E.D. Arthur and M.B. Chadwick, Comprehensive Nuclear Model Calculations: Theory and Use of the GNASH Code, LA-UR-96-3739.

7. D.P. McNabb et al., Uncertainty Budget and efficiency analysis for the ${ }^{239} \mathrm{Pu}(\mathrm{n}, 2 \mathrm{n})$ reaction cross section measurements, UCRL-ID-139906.

8. W. Younes et al., Abs. of Capt. Gamma-ray Symp. Sept. 1999, UCRL-JC-135097 Abs.

9. W. Younes et al., Proceedings of the $10^{\text {th }}$ International Symposium on Capture Gammaray Spectroscopy and Related Topics, Sante Fe, NM, 1999, ed. S. Wender, to be published by AIP, UCRL-JC-135097. 